\title{
Análisis de viabilidad de microrredes eléctricas con alta penetración de recursos renovables en zonas urbanas: caso de estudio condominios residenciales
}

\author{
(Viability of micro electrical networks with high penetration \\ of renewable resources in urban areas: Case study \\ of residential condominiums)
}

Danny Javier Trujillo Sandoval', Fabricio Ismael Mosquera Velásquez², Edwin Marcelo García Torres³

\begin{abstract}
Resumen
El presente estudio aborda la implementación de microrredes eléctricas en zonas urbanas como respuesta a la necesidad de inserción de energías renovables en las sociedades. En una primera fase se caracteriza el perfil de consumo de energía de un condominio con carga de tipo residencial, comercial y comunitaria, abordando la construcción de este perfil mediante levantamiento de cargas reales y criterios estadísticos de factores de uso y coincidencia. En la segunda fase se analizan los recursos naturales velocidad del viento y radiación solar disponibles en la zona de estudio a través del software especializado Homer Pro con coordenadas de latitud y longitud. Posteriormente se analiza y define la viabilidad de los elementos de generación eléctrica renovable por emplear, también se plantea un posible grupo electrógeno y su uso de combustible con el fin de complementar la microrred en aspectos de inercia y estabilidad. Con la demanda de energía y recursos de generación planteados se diseñan alternativas de arquitectura de una potencial microrred para el condominio, se hacen simulaciones para los casos aislado e interconectado con valores reales del entorno y un análisis económico de la implementación, para obtener la viabilidad de la microrred.
\end{abstract}

\section{Palabras clave}

Energía renovable, microrred, fotovoltaico, residencial, eólico.

\begin{abstract}
This study addresses the implementation of electrical micro-grids in urban areas as a response to the need for the insertion of renewable energies in different societies. In a first phase, the energy consumption profile of a condominium with a residential, commercial type load is characterized. and community, addressing the construction of said profile by lifting real loads and statistical criteria of use and coincidence. In the second phase, the natural resources, wind speed and solar radiation available in the study area are analyzed through the specialized software Homer Pro with latitude and longitude coordinates, subsequently the viability of the renewable electricity generation elements is analyzed and defined use, a possible generator set and its use of fuel are also proposed in order to complement the micro-grid in aspects of inertia and stability. With the energy demand and generation resources raised, architectural alternatives of a potential micro-network for the condominium are designed, simulations are carried out for isolated and interconnected cases with real values of the environment and an economic analysis of the implementation, obtaining the viability of the micro network.
\end{abstract}

\section{Keywords}

Renewable energy, micro-grid, photovoltaic, residential, wind. 3614]. 


\section{Introducción}

El desarrollo de las sociedades y sus diferentes elementos conlleva en la actualidad al pensamiento sostenible, con el fin de garantizar el bienestar futuro del ser humano y del medio que lo rodea, recordando que existe un vínculo claro entre el desarrollo institucional del país y el contexto propicio para el desarrollo de energías renovables (Recalde et al., 2015), por lo tanto, la seguridad energética involucra la revisión y vigilancia de todos los países (Umbarila Valencia et al., 2015). Además, la generación de energía eléctrica a través de combustibles fósiles llegará en pocas décadas a su fin, pues se estimó, hace algunos años, que las reservas de petróleo con el ritmo de consumo actual durarán aproximadamente 5 décadas (Khaligh \& Li, 2010). Las fuentes hídricas para producción de energía también han ocasionado inconvenientes como sequías y cambios en el clima (Guacaneme et al., 2018) que cada vez son inciertos debido al cambio climático global. Estos escenarios presentan a las energías renovables como una de las valiosas alternativas (Guacaneme et al., 2018), con tasas de crecimiento promedio en generación renovable al 2018 de 5.3 \% (IEA, 2019), y que contribuirán al bienestar del ser humano y su entorno. Es así que ya en muchas zonas del mundo se utilizan en hogares e industrias las energías a base del viento, radiación solar, biocombustibles, entre otras; y varios organismos y empresas han centrado sus esfuerzos para desarrollar soluciones tecnológicas asociadas.

El desafío de cualquier compañía de servicios energéticos es proporcionar energía limpia y adecuada con una forma conveniente para el consumidor, ya que tiene una relación directa con los estándares de vida, aliviar la pobreza y garantizar la seguridad energética. Los países desarrollados están reduciendo su dependencia del combustible fósil e invierten proactivamente y en montos considerables, en combustibles alternativos para proteger el sistema ecológico para el desarrollo sostenible (Sivarasu et al., 2015; Delboni et al., 2018). Existen a nivel mundial varios ejemplos de líderes como China que en 2018 mostró un valor cercano al 45 \% del total mundial en aumento de la capacidad de electricidad renovable. El segundo mayor crecimiento de capacidad renovable es de la Unión Europea, se debe considerar que, en este escenario, un $85 \%$ del crecimiento fue a partir de energía solar y eólica (International Energy Agency [IEA], 2019).

Las energías renovables con mayor penetración y competitividad a nivel mundial son las eólica y fotovoltaica (IEA, 2019; Beltrán-Telles et al., 2017; Gómez et al., 2018). Estas brindarán una potencia localmente, lo que a su vez disminuirá la corriente que existe a lo largo de la sección del conductor, para así mitigar las pérdidas en el sistema de distribución (Mosquera, 2020), pues ofrecen varias ventajas de implementación, mantenimiento y producción de energía, perfilándose como los grandes aportes renovables del futuro por su naturaleza y abundancia en la actualidad.

Existen varios estudios en los cuales la energía fotovoltaica resulta atractiva a nivel de grupos residenciales dada su naturaleza estática en cuanto a los componentes mecánicos, diversidad de bondades de instalación, costos de mantenimiento, flexibilidad en el control asociado, back up de energía, entre otros (IEA, 2019; Sivarasu et al., 2015; Amrr et al., 2018; Lata-García et al., 2017, October 18-20; Manoj Kumar et al., 2020; Pascual et al., 2014; Rousis et al., 2018). Esto también ha conllevado al estudio más profundo de generación distribuida y microrredes con el fin del futuro energético de las sociedades. 
Las microrredes pueden contemplar modos aislado o interconectado con la red (Pascual et al., 2013, September, 15-19; Rodriguez-Diaz et al., 2017, June 27-29; Yousaf et al, 2019, April 16-17) y se pueden tener múltiples configuraciones con diversidad de recursos energéticos y tipos de carga. Usualmente también se las relaciona con la aplicación de energías renovables, generación distribuida, mejoramiento de eficiencia y reducción de pérdidas en sistemas eléctricos, sistemas de almacenamiento, descentralización de suministros, reducción en emisión de C02, entre otros (Sivarasu et al., 2015; Delboni et al., 2018; Rodriguez-Diaz et al., 2017, June 27-29; Suresh et al., 2020).

Esta investigación plantea la evaluación de recursos renovables a través de software especializado en condominios de tipo residencial que incluyen consumos comunitarios y comerciales, proponiendo también un diseño de una potencial microrred en la zona caso de estudio planteado y evaluándola económicamente como sistema aislado e interconectado. El trabajo en las primeras dos fases describe el condominio residencial, las áreas comunes y comerciales, y caracteriza la demanda de energía a través del estudio independiente de carga residencial por medio de departamentos y locales comerciales standard y consumo comunitario general. En la tercera fase se analizan los recursos de viento y radiación solar de la zona de estudio mediante el software especializado Homer Pro. En la cuarta y quinta fase, con los resultados validados, se diseña, simula y analiza la potencial microrred para el caso de estudio.

\section{Metodología}

\subsection{Diseño de la investigación}

El diseño de la investigación se basó en una metodología mixta secuencial compuesta por dos fases: en la primera fase se aplicó un diseño de investigación cualitativo con alcance descriptivo basado en modalidad documental y de entrevista para caracterizar el condominio, sistema eléctrico y detalles de consumo actual. En la segunda, mediante un diseño de investigación cuantitativo y alcance descriptivo, se construyeron los perfiles de demanda, análisis de recursos energéticos renovables disponibles, diseño de la micro red y análisis de viabilidad financiera de los diferentes escenarios planteados.

\subsection{Zona de estudio y configuración inicial del sistema}

\subsubsection{ZONA DE ESTUDIO}

Los condominios estándar están situados en el sector sur de la capital ecuatoriana. Este condominio es de tipo residencial con 14 departamentos dentro del edificio, con 2 locales comerciales en la planta baja y áreas comunales. En la Figura 1 se puede ver el tipo de edificación sujeto de estudio y su distribución. 
Figura 1. Vistas de la configuración del condominio

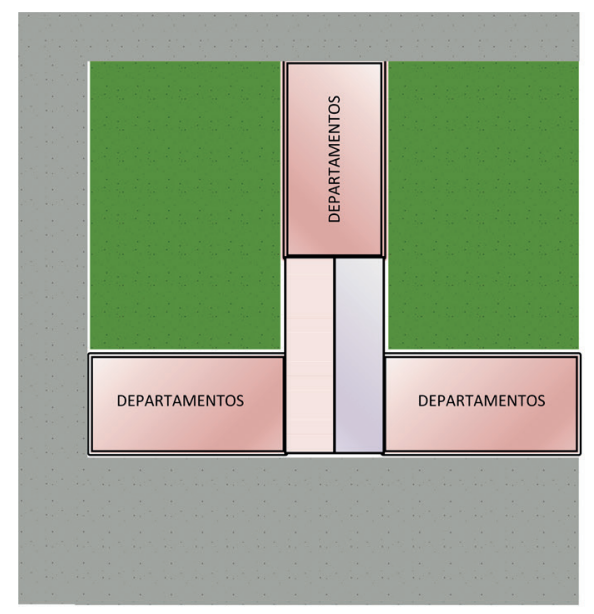

VISTA SUPERIOR

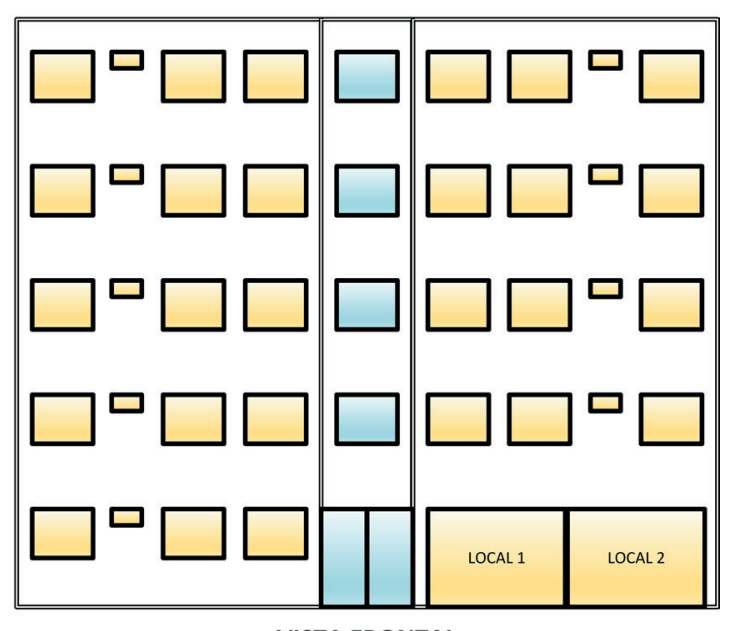

VISTA FRONTAL

\subsubsection{CONFIGURACIÓN INICIAL DEL SISTEMA}

En la Figura 2 se presenta un diagrama eléctrico de tipo esquemático general del condominio, en el cual se identifican los diferentes puntos de conexión en cuanto a tableros principal y secundarios con los cuales se cuenta. Esta información ha sido levantada in situ con el fin de sentar las bases para la sección de estudio de demanda, recursos renovables y diseño de la microrred.

Figura 2. Esquema eléctrico del condominio

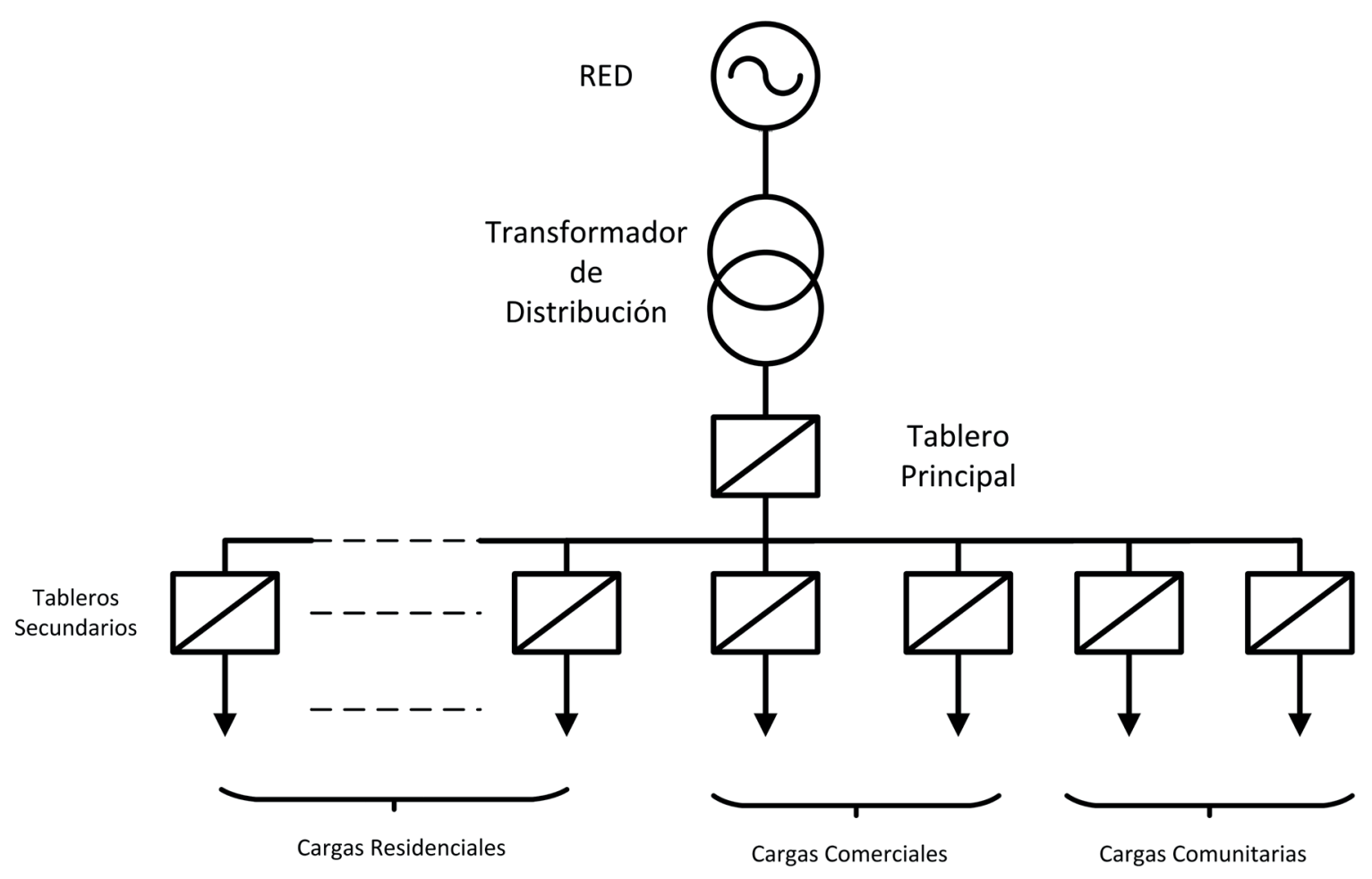




\subsection{Recolección de datos, instrumentos y procesamiento}

Para el estudio se recopilaron datos de la edificación, esquema eléctrico y consumo mediante planillas durante el primer semestre de 2020 en trabajo de campo in situ. Para el tratamiento de los datos e información, se utilizó el software Matlab R2018b y Microsoft Excel 2016; para el caso del análisis técnico económico de la microrred y energías renovables de la zona, se utilizó el software HOMER Pro, Versión 3.13.8. A continuación, se detallan las ecuaciones y fundamentos para el procesamiento de datos y análisis de información de las diferentes secciones del proyecto.

\subsubsection{Perfil de demanda de energía}

La caracterización del perfil de consumo del condominio es clave pues permitirá sentar bases sólidas para diseño y simulación la potencial microrred. En el estudio se han identificado tres tipos de carga: la residencial asociada a departamentos, la comercial asociada a los locales comerciales en planta baja y la comunitaria en la cual se han incluido cargas comunes y de mantenimiento general.

Para el cálculo de demanda del condominio se ha levantado una lista inicial de cargas eléctricas, con sus datos de potencia en Watts, se ha revisado los patrones de consumo en las planillas y entrevistado al administrador del condominio con el fin de establecer criterios que aporten a la investigación. Una vez levantados los datos se han aplicado factores de utilización y coincidencia para calcular la energía consumida diaria y mensual de cada carga de acuerdo con (1) y (2).

$$
\begin{aligned}
& D d=\sum_{i=1}^{24} P_{c} f_{c} f_{u} \\
& D m=\sum_{i=1}^{24} D d
\end{aligned}
$$

Donde:

$D d$ demanda diaria

$D m$ demanda mensual

$P_{c}$ potencia de carga $[k W]$

$f_{c}$ factor de coincidencia

$f_{u}$ factor de utilización

Una vez obtenidos los consumos individuales se consolidan los consumos por tipo de carga a nivel diario y mensual.

\subsubsection{Análisis de ReCURSos Renovables y no ReNovables en LA zona}

A nivel mundial, la capacidad total de electricidad renovable aumentó en 178 GW en 2018 (IEA, 2019), como se muestra en la Figura 3; esto obedece claramente a la necesidad de cambio de las matrices energéticas y a los recursos naturales con los que se cuentan en las zonas de aplicación. 
Figura 3. Capacidad neta anual por tecnología GW
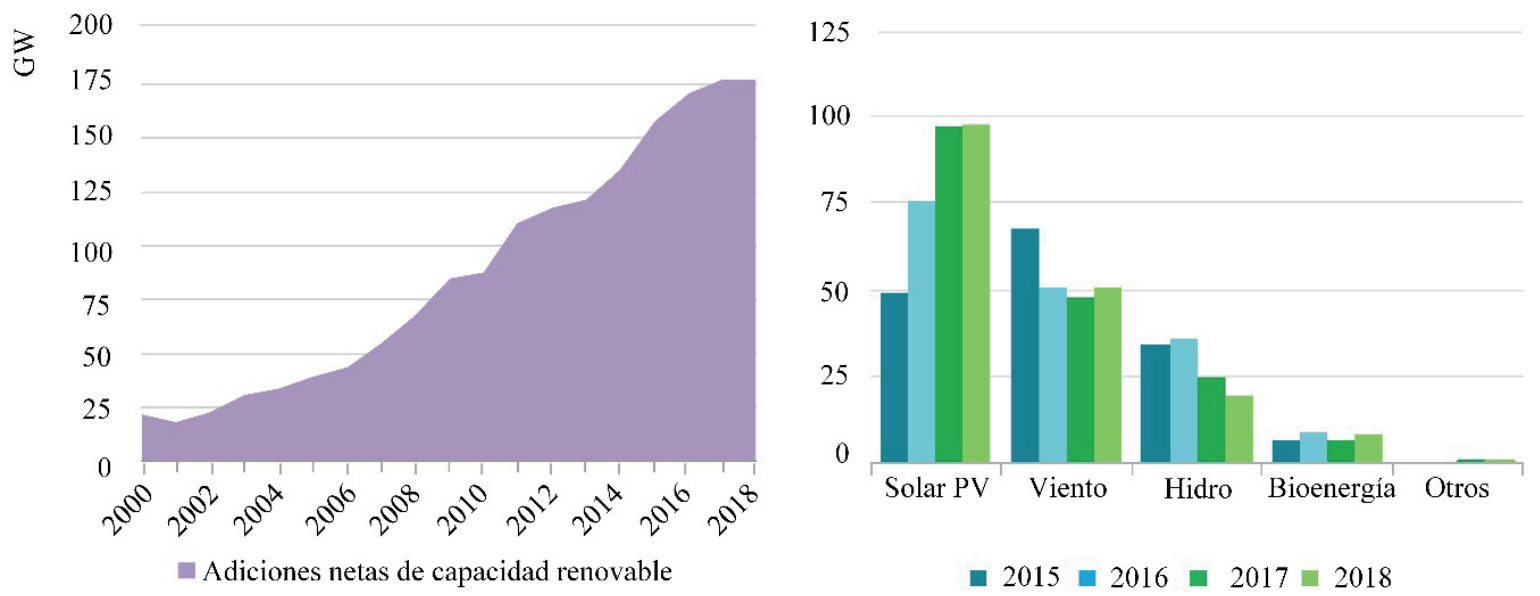

Nota: Tomado de (IEA, 2019).

Al depender directamente de los recursos naturales disponibles en la zona, existen diversidad de arquitecturas y criterios aplicables en la generación de energía eléctrica renovable, sin embargo, las más comunes en aplicaciones residenciales y comerciales son eólico y solar, por lo cual en esta investigación se analizan los recursos de la zona del caso de estudio mediante el software Homer Pro para obtener datos fiables de los recursos viento y radiación solar mediante coordenadas de latitud y longitud.

\subsubsection{Diseño de ARQUiTECTURA dE LA MICRORRED}

Para las diferentes arquitecturas se han empleado elementos como paneles solares, grupos electrógenos, baterías, inversores, entre otros. A continuación, se describen los elementos empleados, así como las ecuaciones que los caracteriza dentro del diseño de la microrred.

Paneles solares: el principal recurso renovable de la microrred propuesta son los paneles fotovoltaicos, que han sido incorporados debido al recurso disponible en la zona de estudio y las bondades de instalación en el condominio.

La potencia para el dimensionamiento del panel solar se realiza en función de la radiación y la temperatura presente en el sector dado. En la ecuación (3) se detalla la potencia del sistema fotovoltaico. Si en esa ecuación no se elige no modelar el efecto de la temperatura en la matriz PV, Homer Pro (Homer Energy, 2019), se asume que el coeficiente de potencia de temperatura es cero, por lo que la ecuación anterior se simplifica en (4):

$$
\begin{aligned}
& P_{P V}=Y_{P V} f_{P V}\left(\frac{\bar{G}_{T}}{\overline{\bar{G}}_{T, S T C}}\right)\left[1+\alpha_{p}\left(T_{c}-T_{c, S T C}\right)\right] \\
& P_{P V}=Y_{P V} f_{P V}\left(\frac{\bar{G}_{T}}{\bar{G}_{T, S T C}}\right)
\end{aligned}
$$


Donde:

$Y_{P V}$ capacidad nominal de la matriz $\mathrm{FV}$, es decir, su potencia de salida en condiciones de prueba [kW]

$f_{P V}$ el factor de reducción de PV [\%]

$\bar{G}_{T}$ radiación solar incidente en el campo $\mathrm{FV}$ en el paso de tiempo actual $\left\lceil\mathrm{kW} / \mathrm{m}^{2}\right\rceil$

$\bar{G}_{T . S T C}$ la radiación incidente en condiciones de prueba estándar $\left\lceil\mathrm{kW} / \mathrm{m}^{2}\right\rceil$

$\alpha_{P}$ el coeficiente de temperatura de potencia $\left[\% /{ }^{\circ} \mathrm{C}\right]$

$T_{c}$ la temperatura de la celda PV en el paso de tiempo actual $\left[{ }^{\circ} \mathrm{C}\right]$

$T_{c, S T C}$ la temperatura de la celda PV en condiciones de prueba estándar $\left[25^{\circ} \mathrm{C}\right]$

Grupo electrógeno: dada la baja inercia que presentan las microrredes en contraste a la generación convencional (Guacaneme et al., 2014), se incluye un grupo electrógeno en base a diésel que permita compensar la inercia y, por lo tanto, controlar frecuencia del sistema, así como compensar necesidad de generación en períodos de escasa producción fotovoltaica y/o baterías.

Baterías: uno de los factores importantes de una batería son sus curvas características que definen en cierta forma el tipo de servicio que se brindará en la aplicación (Trujillo Sandoval \& Torres García, 2020). En el caso del diseño de la microrred son necesarias puesto que se necesita almacenar la energía que se genera durante el día en los paneles fotovoltaicos con el fin de que sea usada en los períodos de escasa o casi nula radiación, por ejemplo, en la noche y madrugada. Las baterías utilizadas son de ion-litio.

Las variables de entrada para el dimensionamiento de las baterías dado en (Homer Energy, 2019) son el cálculo de potencia máxima de carga de la batería, donde (5) proporciona la cantidad máxima de energía que puede ser absorbida por el sistema; la segunda limitación se refiere a la tasa de carga máxima del componente de almacenamiento, su potencia de carga de almacenamiento correspondiente a esta velocidad de carga máxima viene dada por la siguiente ecuación (6), y la tercera limitación (7) se refiere a la corriente de carga máxima del componente de almacenamiento. Homer Pro establece la potencia máxima de carga de almacenamiento igual al menor de estos tres valores, suponiendo que cada uno se aplica después de las pérdidas de carga, por lo tanto, se lo modela en la ecuación (8).

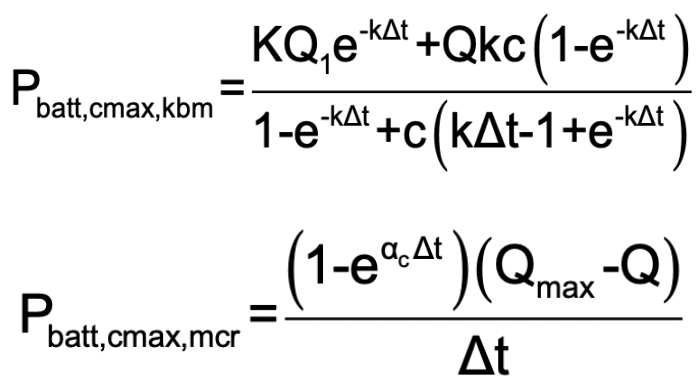




$$
P_{\text {batt, } \text { cmax,mcc }}=\frac{N_{\text {batt }} I_{\text {max }} V_{\text {nom }}}{1000}
$$

$$
P_{\text {batt,cmax }}=\frac{\operatorname{MIN}\left(P_{\text {batt }, c m a x, x b m}, P_{\text {batt,cmax }, \text { mcr }}, P_{\text {batt }, c m a x, m c c}\right)}{\eta_{\text {batt }, c}}
$$

Donde:

$Q_{1}$ la energía disponible $[k W h]$ en el almacenamiento al comienzo del paso del tiempo

$Q$ la cantidad total de energía $[k W h]$ en el almacenamiento al comienzo del paso del tiempo

$C$ la relación de capacidad de almacenamiento

$K$ la tasa de almacenamiento constante $[k-1]$

$\Delta t$ la duración del paso de tiempo $[h]$

$\alpha_{c}$ es la tasa de carga máxima del almacenamiento $[A / A h]$

$Q_{\max }$ es la capacidad total del banco de almacenamiento

$N_{\text {batt }}$ la cantidad de baterías en el banco de almacenamiento

$I_{\text {max }}$ la corriente de carga máxima del almacenamiento $[A]$

$V_{\text {nom }}$ la tensión nominal del almacenamiento $[\mathrm{V}]$

$\eta_{\text {batt }, c}$ eficiencia de la carga de almacenamiento

De acuerdo con el método presentado por Homer Energy (2019) en (9) se establece la potencia máxima de descarga variando de un paso de tiempo a otro según su estado de carga y su historial reciente de carga/descarga que proporciona la cantidad máxima de energía que el banco de almacenamiento puede descargar durante un periodo de tiempo específico. Homer Pro asume que las pérdidas de descarga ocurren después de que la energía abandona el sistema, por lo tanto, la siguiente ecuación (10) proporciona la potencia de descarga máxima del banco de almacenamiento.

$$
\begin{gathered}
P_{\text {batt,cmax,kbm }}=\frac{-k c Q_{\max }+K Q_{1} e^{-k \Delta t}+Q k c\left(1-e^{-k \Delta t}\right)}{1-e^{-\kappa \Delta t}+c\left(k \Delta t-1+e^{-\star \Delta t}\right)} \\
P_{\text {batt,dmax }}=\eta_{\text {batt,d }} P_{\text {batt,dmax }, k b m}
\end{gathered}
$$

Donde:

$\eta_{b a t t, d}$ eficiencia de la descarga de almacenamiento 
Inversor DC/AC: utilizado en el proyecto para convertir la energía de paneles solares y de baterías, y poder conectar al tablero de distribución principal que alimenta a sus respectivas ramificaciones.

El convertidor es un dispositivo que convierte la energía eléctrica de CC a CA en un proceso llamado inversión, y de CA a CC en un proceso llamado rectificación. El tamaño del convertidor, que es una variable de decisión, se refiere a la capacidad del inversor, es decir, la cantidad máxima de energía de CA que el dispositivo puede producir al invertir la energía de CC (Homer Energy, 2019).

\subsubsection{Simulación de la microrred en Homer Pro}

Para simular los diferentes escenarios, se ha utilizado el software especializado Homer Pro. La microrred general y demandas eléctricas planteadas se muestran en la Figura 4. Cabe señalar que para cada arquitectura se ha modificado el ingreso o egreso del grupo electrógeno y de la red como se podrá analizar en secciones posteriores.

Figura 4. Arquitectura del modelo de la microrred
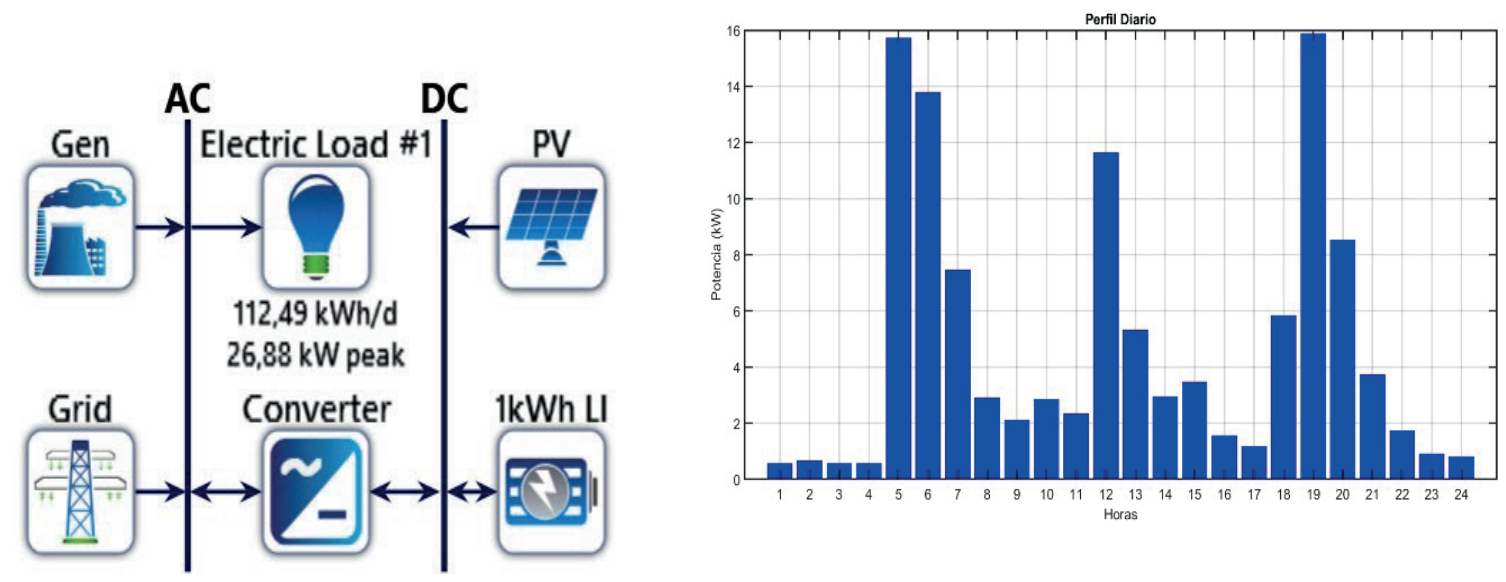

En las tablas 1 y 2 se muestran los valores ingresados en Homer Pro para cada elemento de la microrred, los cuales son la base para la simulación de escenarios. Cabe señalar que estos valores son referenciales al mercado de la zona de estudio y al año 2020.

Tabla 1. Datos de ingreso del caso de estudio

\begin{tabular}{|c|c|c|c|c|}
\hline Elemento & Capital (\$/kW) & $\begin{array}{c}\text { Costo de reemplazo } \\
(\mathbf{\$} / \mathbf{k W})\end{array}$ & $\begin{array}{c}\text { Costo de O\&M } \\
\text { (\$/año) }\end{array}$ & Vida útil (años) \\
\hline PV & 700 & 700 & 10 & 25 \\
\hline Baterías & 469 & 450 & 10 & 10 \\
\hline Convertidor & 250 & 250 & 0 & 10 \\
\hline
\end{tabular}


Tabla 2. Datos de ingreso del caso de estudio-GE

\begin{tabular}{|c|c|c|c|c|c|}
\hline Elemento & Capital $\mathbf{( \$ / k W )}$ & $\begin{array}{c}\text { Costo de } \\
\text { reemplazo } \\
(\mathbf{\$} / \mathbf{k W})\end{array}$ & $\begin{array}{c}\text { Costo de O\&M } \\
\mathbf{( \$ / h - o p )}\end{array}$ & $\begin{array}{c}\text { Vida útil } \\
\text { (horas) }\end{array}$ & $\begin{array}{c}\text { Precio del } \\
\text { combustible } \\
(\mathbf{\$} / \mathbf{L})\end{array}$ \\
\hline Grupo electrógeno & 280 & 250 & 0.02 & 15000 & 0.9 \\
\hline
\end{tabular}

En los escenarios en los cuales se incluye la red eléctrica el precio de la energía eléctrica ha sido colocado en 0.09 (\$/kWh). En la siguiente sección se establecerán los parámetros de dimensionamiento para los elementos mencionados, así como criterios resultantes de los escenarios planteados.

\subsubsection{Modelo económico en Homer Pro}

La economía juega un papel integral en el proceso de simulación de Homer Pro, en el que opera el sistema para minimizar el costo presente neto total (Rousis et al., 2018; Homer Energy, 2019). Las fuentes renovables tienden a tener altos costos iniciales de capital y bajos costos operativos, mientras que las fuentes convencionales no renovables tienden a tener bajos costos de capital inicial y altos costos operativos. Homer Pro utiliza el costo presente neto total (NPC) para representar el costo del ciclo de vida de un sistema. El NPC total condensa todos los costos e ingresos que ocurren dentro de la vida útil del proyecto en una suma global en dólares de hoy, con futuros flujos de efectivo descontados al presente utilizando la tasa de descuento. Además, entre muchos parámetros, el modelador especifica la tasa de descuento, inflación y la vida útil del proyecto (Homer Energy, 2019).

Todos los costos en Homer Pro son reales, lo que significa que se definen en términos de dólares constantes. Para cada componente del sistema, el modelador especifica el costo de capital inicial, que ocurre en el año cero; el de reemplazo, que ocurre cada vez que el componente necesita reemplazo al final de su vida útil, y el de operación y mantenimiento, que ocurre cada año del proyecto de por vida. El usuario especifica la vida útil de la mayoría de los componentes en años, pero Homer Pro calcula la vida útil de la batería y los generadores (Homer Energy, 2019).

Homer Pro (Homer Energy, 2019) utiliza las siguientes ecuaciones:

$$
\begin{aligned}
& C_{n p c}=\frac{C_{\text {ann,tot }}}{\operatorname{CRF}\left(i, R_{\text {proj }}\right)} \\
& \operatorname{CRF}(i, N)=\frac{i(1+i)^{N}}{(1+i)^{N}-1} \\
& C O E=\frac{C_{\text {ann,tot }}}{E_{\text {prim }}+E_{\text {def }}+E_{\text {grid,sales }}}
\end{aligned}
$$

Donde:

$C_{N P C}$ costo presente neto total

i la tasa de interés real anual (la tasa de descuento) 
$C_{\text {ann,tot }}$ costo total anualizado

$R_{\text {proj }}$ tiempo de vida del proyecto

CRF(.) factor de recuperación de capital

N número de años

COE el costo nivelado de energía

$E_{\text {prim }}$ cantidades totales de carga por año

$E_{\text {def }}$ cantidades de carga diferible por año

$E_{\text {grid,sales }}$ cantidad de energía vendida por año

\section{Resultados y discusión}

\subsection{Caracterización del perfil de demanda de energía}

Con el procedimiento descrito en la sección 2.3.1, se obtienen las curvas diarias de demanda de energía resultantes del condominio, como se observa en la Figura 5, en las que se diferencia el tipo de consumo y se logra el primer insumo para la simulación del sistema en Homer Pro.

Figura 5. Curvas del consumo del condominio
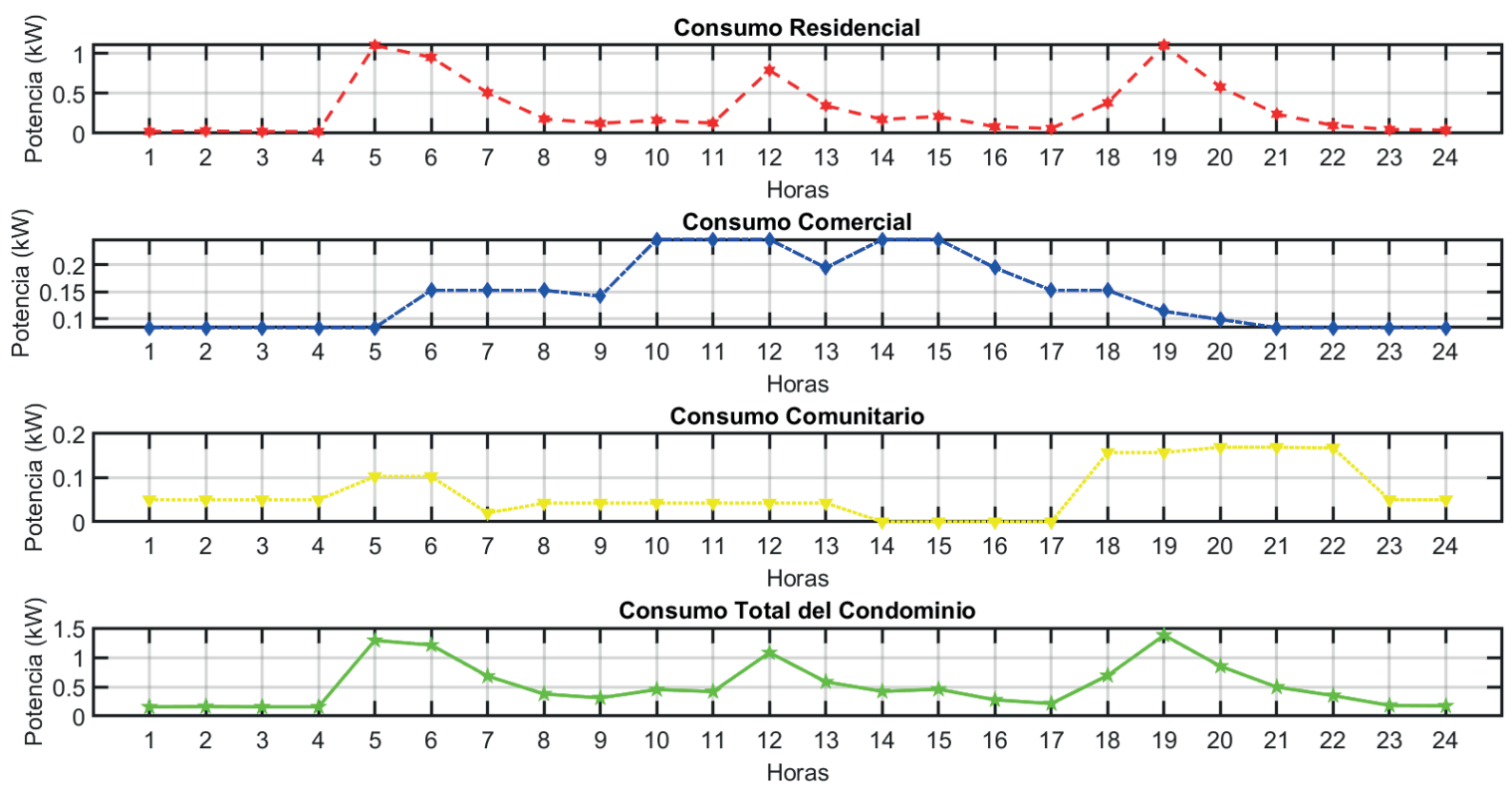

En la tabla 3 se desglosan los valores de demanda de energía diaria y mensual para el consumo individual y del condominio en general con el fin de agregar valor el momento de su aplicación y toma de decisión para el diseño de la microrred eléctrica en secciones posteriores. 
Tabla 3. Consumos de energía

\begin{tabular}{|c|c|c|c|c|}
\cline { 2 - 5 } \multicolumn{1}{c|}{} & \multicolumn{3}{c|}{ Consumo de energía eléctrica } \\
\cline { 2 - 5 } & \multicolumn{2}{c|}{ Individual } & Condominio \\
\hline Carga & kWh/dia & kWh/Mes & 103.88 & kWh/Mes \\
\hline Residencial & 7.42 & 222.61 & 7.00 & 210.00 \\
\hline Comercial & 3.50 & 105.00 & 1.60 & 48.06 \\
\hline Comunitario & - & - & 112.49 & 3374.59 \\
\hline Total & 10.92 & 327.61 & & \\
\hline
\end{tabular}

\subsection{Análisis de recursos renovables y no renovables en la zona}

En esta sección se analizan los recursos naturales velocidad del viento y radiación solar disponibles en la zona de estudio, a través del software especializado Homer Pro con coordenadas de latitud y longitud para recopilar los datos deseados. En la Figura 6 se puede ver el recurso de radiación solar con el que se cuenta en la zona de estudio, lo que resulta adecuado pues representa un valor superior a $4\left(\mathrm{kWh} / \mathrm{m}^{\wedge} 2 /\right.$ día) que en posteriores secciones podrá cubrir una parte importante del consumo diario de los departamentos y/o del condominio en general.

Figura 6. Radiación e índice de claridad en las coordenadas de la zona estudio

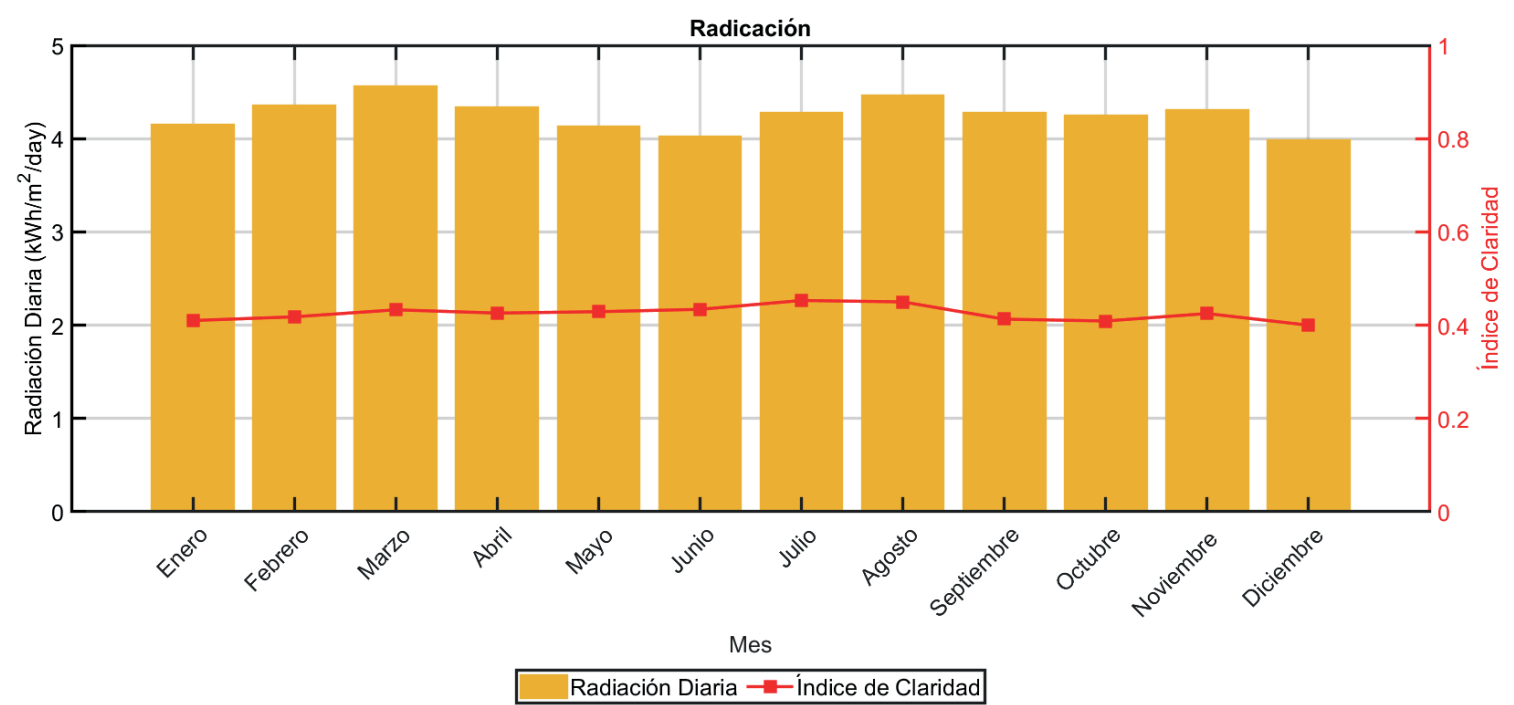

En la Figura 7 se observan los valores del recurso viento tomados de Homer Pro, los cuales tienen una media anual de $2.34(\mathrm{~m} / \mathrm{s})$, lo que refleja que, para este estudio, no son valores adecuados, pues se estima que para proyectos eólicos se debe contar con valores superiores a 3 a 4 (m/s) (EOLICCAT, 2020). Por este motivo, se descarta el uso de aerogeneradores en la zona y se diseña la microrred con energía fotovoltaica, grupos de baterías y un grupo electrógeno con el fin de garantizar la inercia de la microrred y una respuesta de frecuencia debido a las variaciones de carga. 
Figura 7. Viento promedio en las coordenadas del caso de estudio

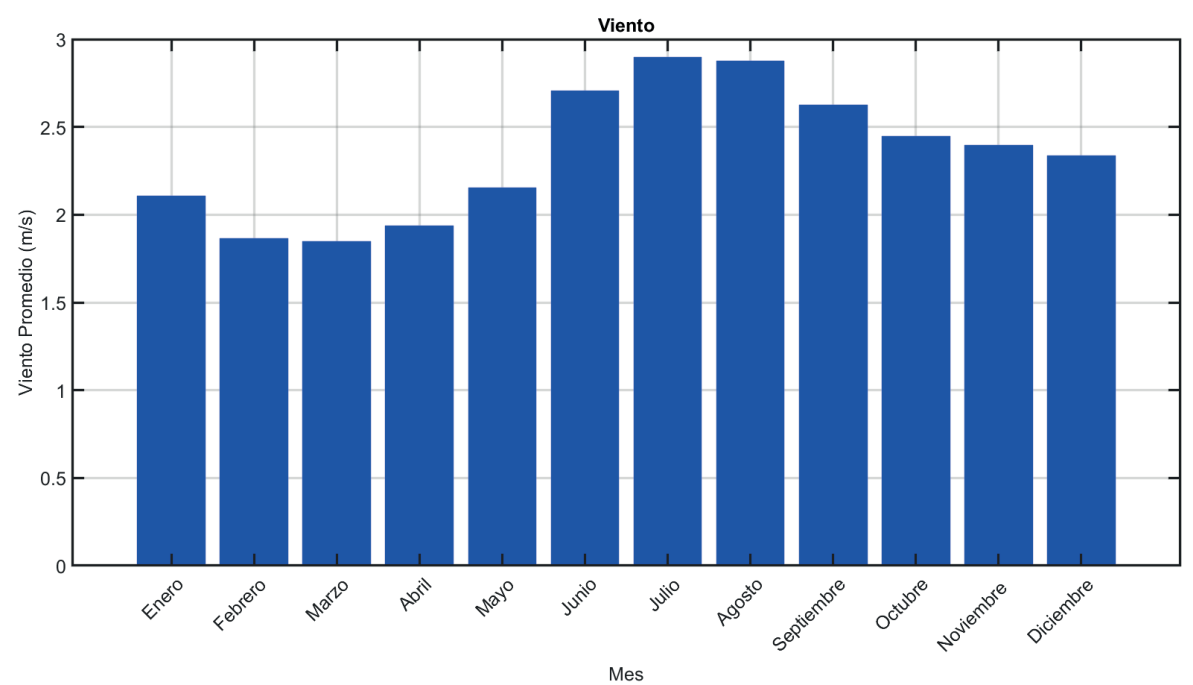

\subsection{Diseño de la arquitectura de la microrred}

Con base en los datos obtenidos de demanda de energía eléctrica del condominio y condiciones de radiación solar y viento de la zona, se diseña la microrred aislada con paneles solares, grupo de baterías y un grupo electrógeno como elementos de generación que incluyen los tres tipos de cargas con las cuales cuenta el condominio. Cabe señalar que también se diseña una posible microrred aislada sin grupo electrógeno para analizar la falta de inercia e incremento de costos que conllevaría esta arquitectura. Como caso especial, se analizará la conexión de la microrred con la red eléctrica principal en la sección de simulación para establecer competencia en cuanto a costos de energía e implementación de la microrred. En la Figura 8 se pueden observar los diagramas de las tres configuraciones diseñadas para el estudio.

Figura 8. Diagrama de las configuraciones de la microrred

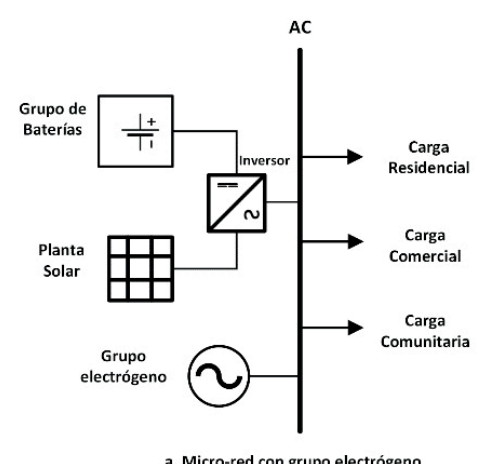

a. Micro-red con grupo electrógeno

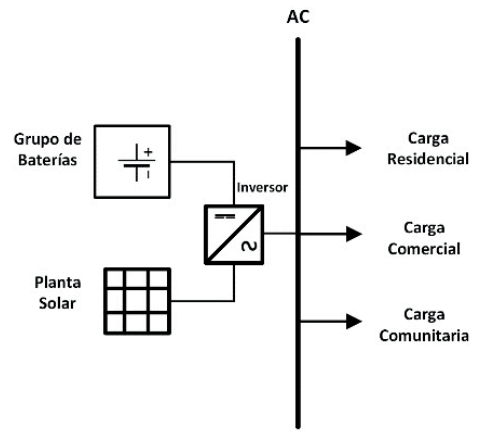

b. Micro-red sin grupo electrógeno

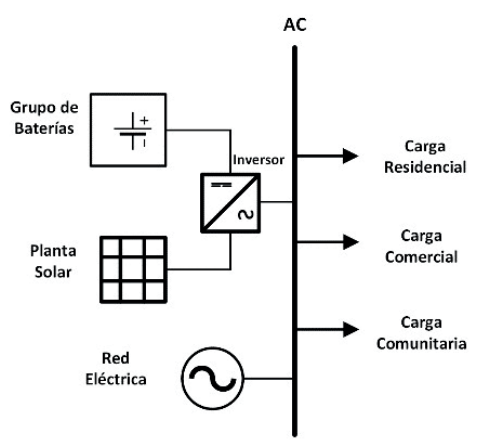

c. Micro-red conectada a la red

Como se menciona anteriormente, se han modelado tres arquitecturas: aislada con grupo electrógeno, aislada sin grupo electrógeno y conectada a la red eléctrica, por lo tanto, se obtienen los resultados presentados en las siguientes secciones. 


\subsection{Microrred aislada}

Estas dos primeras arquitecturas se desagregan en 4 escenarios, debido a que cada diseño de la microrred se analiza en su mejor y peor condición. Cabe resaltar que todos los elementos se han parametrizado para correr el optimizador de Homer Pro, ninguno ha sido forzado mediante valores o escalas fijas. En la tabla 4 se pueden obtener los valores finales para los diferentes elementos de la microrred planteada.

De acuerdo con la simulación, los valores óptimos de dimensionamiento para la planta fotovoltaica y baterías superan el $50 \%$ del total requerido por la microrred en cuanto a potencia instalada en la arquitectura 1, y son el $100 \%$ en la arquitectura 2, en cuanto a despacho. En la tabla 5 se puede observar que los diferentes escenarios muestran desde el $24 \%$ hasta el $100 \%$ de participación de energía renovable garantizando un valor significativo en la penetración de este tipo de energía en el sistema.

Tabla 4. Arquitectura de los casos de estudio

\begin{tabular}{|c|c|c|c|c|c|}
\hline & & Arqui & tura 1 & Arquit & ura 2 \\
\hline & Escenarios & 1 & 2 & 3 & 4 \\
\hline \multirow{4}{*}{ Elementos utilizados } & PV & & & & \\
\hline & GEN & & & & \\
\hline & Grupo de baterías & & & & \\
\hline & Convertidor & & & & \\
\hline \multirow{4}{*}{$\begin{array}{l}\text { Dimensionamiento } \\
\text { de elementos } \\
\text { de la microrred }\end{array}$} & $\mathrm{PV}(\mathrm{kW})$ & 15 & 113 & 173 & 339 \\
\hline & Gen(kW) & 30 & 30 & & \\
\hline & Baterías Li-Ion (kW) & 32 & 141 & 193 & 423 \\
\hline & Convertidor(kW) & 13.6 & 13.7 & 73.9 & 123 \\
\hline
\end{tabular}

Tabla 5. Escenarios de los casos de estudio

\begin{tabular}{|c|c|c|c|c|c|}
\hline & Escenarios & 1 & 2 & 3 & 4 \\
\hline \multirow{4}{*}{ Componentes } & PV & & & & \\
\hline & GEN & & & & \\
\hline & Grupo de baterías & & & & \\
\hline & Convertidor & & & & \\
\hline \multirow{4}{*}{ Costo } & NPC (\$) & $\$ 183.52$ & $\$ 319.69$ & $\$ 406.16$ & $\$ 824.71$ \\
\hline & COE $(\$)$ & $\$ 0.38$ & $\$ 0.67$ & $\$ 0.85$ & $\$ 1.72$ \\
\hline & Costo de operación (\$) & $\$ 12.51$ & $\$ 13.92$ & $\$ 15.06$ & $\$ 30.65$ \\
\hline & Capital inicial (\$/año) & $\$ 37.32$ & $\$ 157.05$ & $\$ 230.20$ & $\$ 466.44$ \\
\hline \multirow{2}{*}{ Sistema } & Fracción renovable (\%) & 24 & 80.6 & 100 & 100 \\
\hline & Total fuel (L/año) & 9.697 & 3.614 & 0 & 0 \\
\hline
\end{tabular}

Como se puede ver en las dos arquitecturas, el incremento de producción de energía fotovoltaica en los sistemas incrementa el costo de la energía, el costo presente neto, el costo de operación y el capital inicial. Asimismo, es necesario señalar que la arquitectura 1, la cual contiene el grupo electrógeno, resulta mejor en cuanto a costos y a viabilidad dado el aporte del inercia al sistema, y tomando en cuenta que en la actualidad el valor del combustible resulta muy competitivo frente a las energías renovables; sin embargo, en un futuro, la escases del recurso conllevará a un cambio paulatino de este escenario, así como la afectación al medio ambiente. 
En la Figura 9 se muestra la arquitectura final para la potencial microrred en la zona con los valores de dimensionamiento de los diferentes elementos.

Figura 9. Diseño de la potencial microrred

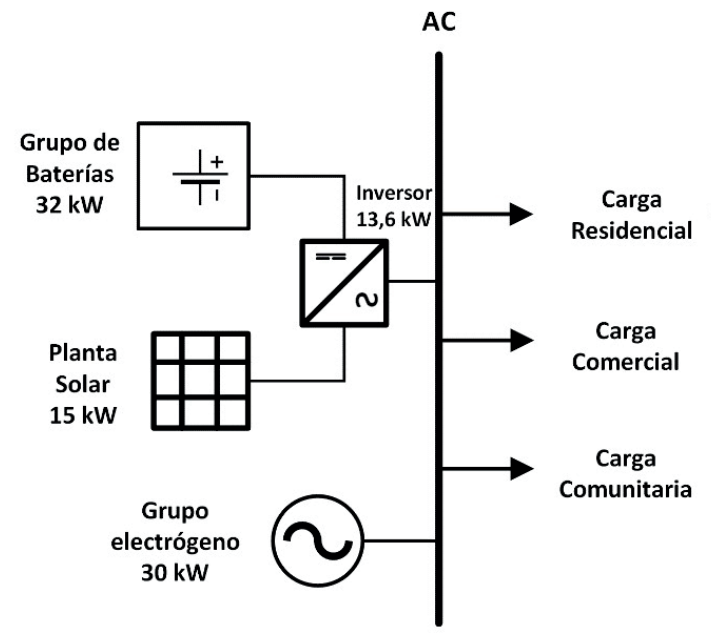

a. Micro-red con grupo electrógeno

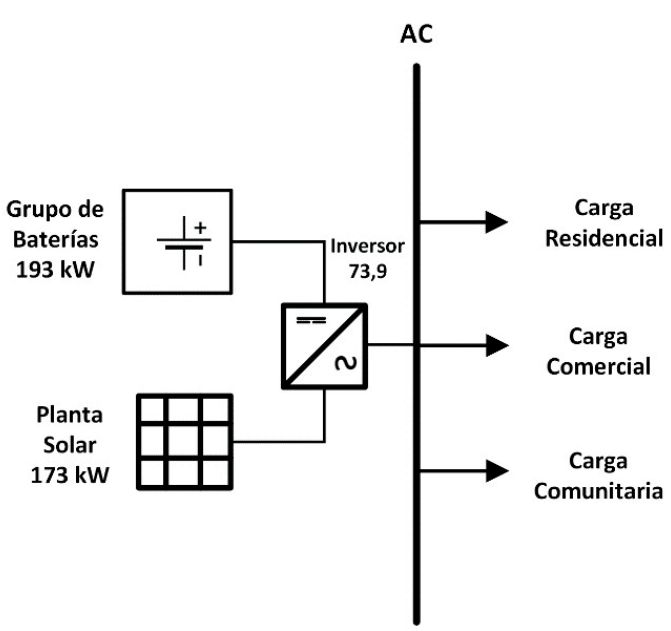

b. Micro-red sin grupo electrógeno

\subsection{Microrred interconectada}

Con el fin de analizar el estado actual de competencia de una microrred vs la red eléctrica convencional con valores económicos reales en la zona, se simula ese escenario en Homer Pro. Al hacer la simulación debido al precio de $0.09(\$ / \mathrm{kWh})$ de la energía de la red los escenarios óptimos son con $100 \%$ de producción de la red eléctrica, pero se debe recordar que Homer Pro ofrece varias alternativas de arquitectura, por lo cual se ha escogido una arquitectura que contemple paneles solares y baterías, y analizado los escenarios de mínimo y máximo aporte de los paneles. En la tabla 6 se presentan los valores resultado de la simulación.

Tabla 6. Microrred interconectada

\begin{tabular}{|c|c|c|c|}
\hline & Escenarios & 1 & 2 \\
\hline \multirow{4}{*}{ Componentes } & PV & & \\
\hline & Grupo de baterías & & \\
\hline & Red & & \\
\hline & Convertidor & & \\
\hline \multirow{4}{*}{ Arquitectura } & $\mathrm{PV}(\mathrm{kW})$ & 0.778 & 113 \\
\hline & Baterías & 1 & 141 \\
\hline & Red & 999.999 & 999.999 \\
\hline & Convertidor(kW) & 0.0722 & 13.7 \\
\hline \multirow{4}{*}{ Costo } & NPC (\$) & $\$ 44.74$ & $\$ 271.91$ \\
\hline & COE (\$) & $\$ 0.09$ & $\$ 0.32$ \\
\hline & Costo de operación (\$) & $\$ 3.74$ & $\$ 10.55$ \\
\hline & Capital inicial (\$/año) & $\$ 1.03$ & $\$ 148.65$ \\
\hline Sistema & Fracción renovable (\%) & 0.651 & 65.4 \\
\hline
\end{tabular}


Se puede ver que el dimensionamiento de elementos del escenario óptimo en esta arquitectura restringe la potencia instalada de paneles solares a menos del $1 \%$, y el costo de la energía básicamente es marcado por la red eléctrica. Al revisar el escenario con alta penetración de energía solar en esta arquitectura, se encuentran costos excediendo el triple del costo de energía de la red y costos presentes netos que permiten concluir la no factibilidad actual de este tipo de microrredes frente a la red desde la perspectiva económica. No obstante, se debe recordar que las microrredes serán una valiosa alternativa en décadas futuras cuando se incrementen los precios del combustible y que, debido a su generación renovable, serán el futuro que permita recuperar y sostener el medio ambiente mundial.

\section{Conclusiones y recomendaciones}

La demanda de energía de un condominio consta de cargas de diferente naturaleza residencial, comercial y comunitaria, por este motivo, la cuantificación tanto en potencia como en el horario de uso y factores de coincidencia y utilización deben ser tratados de forma independiente para lograr una curva de perfil de demanda adecuada.

En el análisis de recursos naturales de la zona de estudio se identificó que la radicación solar promedio está por sobre los 4 ( $\mathrm{kWh} / \mathrm{m}^{\wedge} 2 /$ día), lo cual es adecuado para el aporte de energía solar de este proyecto. En el caso del viento, se observó que las velocidades del este son menores a $4(\mathrm{~m} / \mathrm{s})$, por lo cual por las características mecánicas de los aerogeneradores no se cuenta con factibilidad para aplicar este recurso en el proyecto de microrred. Cabe señalar que el análisis de recursos naturales para aplicación en microrredes es independiente en cada zona, pues contempla variables de diferente naturaleza.

Al diseñar y simular la microrred aislada se determinó que el modelo con grupo electrógeno resulta mejor en todos los costos asociados, además, en la perspectiva técnica se cuenta con aporte de inercia a la microrred; sin embargo, al ser fuente de generación no renovable impacta en el ambiente por la emisión de gases. Dentro de la configuración aislada, al simular sin el grupo electrógeno se evidencia el incremento de los costos de energía en un 121.3 \%; costo presente neto en $123.7 \%$; de operación en 20.4 \% y capital inicial 516.8 \%. Estos incrementos obedecen al total aporte de energía renovable al $100 \%$. En la simulación de la microrred interconectada con la red eléctrica se puede ver la no factibilidad de esta desde el punto de vista económico, pues la optimización de Homer Pro siempre prioriza a la red por su bajo costo (0.09 $\$ / \mathrm{kWh}$ ) frente a las energías renovables en la actualidad de la zona de estudio, por tal motivo, el aporta de energía renovable es menor al $1 \%$ en el sistema.

\section{Referencias}

Amrr, S. M., Alam, M. S., Asghar, M. S. J., \& Ahmad, F. (2018). Low cost residential microgrid system based home to grid (H2G) back up power management. Sustainable Cities and Society, 36(January), 204-214. https://doi.org/10.1016/j.scs.2017.10.016

Beltrán-Telles, A., Morera-Hernández, M., López-Monteagudo, F. E., \& Villela-Varela, R. (2017). Prospectiva de las energías eólica y solar fotovoltaica en la producción de energía eléctrica. CienciaUAT, 11(2), 105. https://doi.org/10.29059/cienciauat.v11i2.742

Delboni, L. F. N., Marujo, D., Balestrassi, P. P., \& Oliveira, D. Q. (2018). Electrical Power Systems: Evolution from Traditional Configuration to Distributed Generation and Microgrids. En Zambroni de Sou- 
za A., \& Castilla, M (Eds.), Microgrids Design and Implementation (1-25). Springer. https://doi. org/10.1007/978-3-319-98687-6_1

EOLICCAT. (2020). Preguntas frecuentes. http://eoliccat.net/preguntas-frecuentes/?lang=es

Gómez, V. A., Hernández, C., \& Rivas, E. (2018). La influencia de los niveles de penetración de la generación distribuida en los mercados energéticos. Información Tecnológica, 29(1), 117-128. https://doi. org/10.4067/S0718-07642018000100013

Guacaneme, J. A., Velasco, D., \& Trujillo, C. L. (2014). Revisión de las características de sistemas de almacenamiento de energía para aplicaciones en micro redes. Información Tecnológica, 25(2), 175-188. https://doi.org/10.4067/S0718-07642014000200020

Guacaneme, W., Rodríguez, A. F., Gómez, L. M., Santamaría, F., \& Trujillo, C. (2018). Desarrollo de un prototipo de micro-red residencial a baja escala. TecnoLógicas, 21(43), 107-125. https://doi. org/10.22430/22565337.1065

Homer Energy. (2019). HOMER PRO: Manual Homer Energy, 1-241. http://www.homerenergy.com/pdf/ HOMERHelpManual.pdf

International Energy Agency. (2019). Renewables 2019 Analysis and forecast to 2024 Explore: Renewables 2019. https://www.iea.org/data-and-statistics/charts/renewable-capacity-growth-bycountry-region-2018-2024

Khaligh, A., \& Li, Z. (2010). Battery, Ultracapacitor, Fuel Cell, and Hybrid Energy Storage Systems for Electric, Hybrid Electric, Fuel Cell, and Plug-In Hybrid Electric Vehicles: State of the Art. IEEE Transactions on Vehicular Technology, 59(6), 2806-2814. https://doi.org/10.1109/TVT.2010.2047877

Lata-García, J., Reyes-Lopez, C., Jurado, F., Fernández-Ramírez, L. M., \& Sanchez, H. (2017, October 18-20). Sizing Optimization of A Small Hydro/Photovoltaic Hybrid System for Electricity Generation in Santay Island, Ecuador By Two Methods. [Proceedings], 1-6. 2017 CHILEAN Conference on Electrical, Electronics Engineering, Information and Communication Technologies, CHILECON 2017, Pucon, Chile. https://doi.org/10.1109/CHILECON.2017.8229539

Manoj Kumar, N., Chopra, S. S., Chand, A. A., Elavarasan, R. M., \& Shafiullah, G. M. (2020). Hybrid Renewable Energy Microgrid for A Residential Community: A Techno-Economic and Environmental Perspective in The Context Of The SDG7. Sustainability, 12(10), 1-30. https://doi.org/10.3390/SU12103944

Mosquera, F. (2020). Localización óptima de plantas virtuales de generación en sistemas eléctricos de potencia basados en flujos óptimos de potencia. Revista de I+D Tecnológico, 16(2), s. p. https:// doi.org/10.33412/idt.v16.2.2827

Pascual, J., Martín, I. S., Ursúa, A., Sanchis, P., \& Marroyo, L. (2013, September, 15-19). Implementation and Control of A Residential Microgrid Based on Renewable Energy Sources, Hybrid Storage Systems and Thermal Controllable Loads, 2304-2309. 2013 IEEE Energy Conversion Congress and Exposition, ECCE 2013, Denver, CO, Estados Unidos. https://doi.org/10.1109/ECCE.2013.6646995

Pascual, J., Sanchis, P., \& Marroyo, L. (2014). Implementation and Control of A Residential Electrothermal Microgrid Based on Renewable Energies, A Hybrid Storage System and Demand Side Management. Energies, 7(1), 210-237. https://doi.org/10.3390/en7010210

Recalde, M. Y., Bouille, D. H., \& Girardin, L. O. (2015). Limitaciones para el desarrollo de energías renovables en Argentina. Problemas del Desarrollo, 46(183), 89-115. https://doi.org/10.1016/j. rpd.2015.10.005

Rodriguez-Diaz, E., Palacios-Garcia, E. J., Anvari-Moghaddam, A., Vasquez, J. C., \& Guerrero, J. M. (2017, June 27-29). Real-time Energy Management System for a hybrid AC/DC residential microgrid, 256-261. 2017 IEEE 2nd International Conference on Direct Current Microgrids, ICDCM 2017, Nuremburg. https://doi.org/10.1109/ICDCM.2017.8001053

Rousis, A. O., Tzelepis, D., Konstantelos, I., Booth, C., \& Strbac, G. (2018). Design of A Hybrid AC/DC Microgrid Using Homer Pro: Case Study on An Islanded Residential Application. Inventions, 3(3), 1-14. https://doi.org/10.3390/inventions3030055 
Sivarasu, S. R., Chandira Sekaran, E., \& Karthik, P. (2015). Development of Renewable Energy Based Microgrid Project Implementations for Residential Consumers in India: Scope, Challenges and Possibilities. Renewable and Sustainable Energy Reviews, 50, 256-269. https://doi.org/10.1016/j. rser.2015.04.118

Suresh, V., Muralidhar, M., \& Kiranmayi, R. (2020). Modelling and Optimization of An Off-Grid Hybrid Renewable Energy System for Electrification in A Rural Areas. Energy Reports, 6, 594-604. https://doi. org/10.1016/j.egyr.2020.01.013

Trujillo Sandoval, D. J., \& Torres García, M. E. (2020). Respuesta de demanda de energía por introducción de vehículos eléctricos: Estado del arte [Response of Demand for Energy by Electric Vehicles Introduction: State of the Art]. Revisa de I+D Tecnológico, 16(1), s. p. https://doi.org/10.33412/ idt.v16.1.2433

Umbarila Valencia, L. P., Alfonso Moreno, F. L., \& Rivera Rodríguez, J. C. (2015). Importancia de las energías renovables en la seguridad energética y su relación con el crecimiento económico. Revista de Investigación Agraria y Ambiental, 6(2), 231. https://doi.org/10.22490/21456453.1419

Yousaf, A., Khan, B. A., Bashir, U., \& Ahmad, F. (2019, April 16-17). Overview of Implementing Microgrid, Its Policies, Incentives and Challenges in Pakistan. [Proceedings], 6-11. 2019 6th International Conference on Electrical and Electronics Engineering, ICEEE 2019, Instabul, Turkey. https://doi. org/10.1109/ICEEE2019.2019.00009 